\title{
LA RESPONSABILIDAD SOCIAL EMPRESARIAL FRENTE AL RETO DE LA COVID-19
}

\section{CORPORATE SOCIAL RESPONSIBILITY FACING THE CHALLENGE OF COVID-19}

\author{
Dr. Jorge Antonio García Gálvez ${ }^{a}$ \\ Dra. Tamara Morales Owseykoff ${ }^{\text {b }}$ \\ Dr. Héctor Rogelio Olivares Galván ${ }^{\text {c }}$
}

\begin{abstract}
${ }^{\text {a } U n i v e r s i d a d ~ V e r a c r u z a n a ~}$ Instituto de Investigaciones en Contaduría, jorgarcia@uv.mx

${ }^{\mathrm{b}}$ Universidad Veracruzana Instituto de Investigaciones en Contaduría, tamorales@uv.mx

${ }^{\mathrm{c}}$ Universidad Veracruzana Instituto de Investigaciones en Contaduría, holivares@uv.mx
\end{abstract}

\section{RESUMEN}

Actualmente la sociedad se encuentra ante una crisis global causada por la pandemia de la COVID-19. Ante la pregunta de cómo hacer sostenible la recuperación de la economía mundial, se presentan opciones que ahora son posibles gracias a los cambios generados por la pandemia. Para las empresas, los programas de Responsabilidad Social Empresarial (RSE) serán útiles para reorientar los modelos de trabajo.

El objetivo del trabajo es analizar los retos y oportunidades que tendrán las empresas en la nueva realidad post-COVID-19 y el papel que puede desempeñar la RSE para ayudar a las empresas a sobrevivir en el futuro inmediato y prosperar a largo plazo.

Este trabajo es cualitativo, utilizándose textos de investigación, medios electrónicos, revistas especializadas en negocios y salud, diarios de circulación nacional e internacional.

Recapitulando, no será posible el retorno a la situación previa a la pandemia. La crisis abre oportunidades para apoyar políticas sostenibles con el medio ambiente y que promuevan los derechos humanos y laborales. La demanda de los consumidores, las redes de proveedores de las empresas, sus fuentes de efectivo, usos de financiamiento y liquidez han cambiado. 
Las empresas pueden encontrar en los programas de RSE herramientas útiles para adaptarse a la nueva realidad.

PALABRAS CLAVE: Responsabilidad Social Empresarial; Pacto Mundial; COVID-19; Cambio estructural.

\section{ABSTRACT}

Society is currently facing a global crisis caused by the COVID-19 pandemic. Faced with the question of how to make the recovery of the world economy sustainable, options are presented that are now possible thanks to the changes generated by the pandemic. For companies, the Corporate Social Responsibility (CSR) programs will be useful to reorient work models.

The objective of this paper is to analyze the challenges and opportunities that companies will have in the new post-COVID-19 reality and the role that CSR can play in helping companies survive in the immediate future and prosper in the long term.

This work is qualitative, using research texts, electronic media, specialized business and health magazines, national and international newspapers.

Recapitulating, a return to the pre-pandemic situation will not be possible. The crisis opens opportunities to support policies that are sustainable with the environment and that promote human and labor rights. Consumer demand, companies' supplier networks, their sources of cash, financing, and liquidity have changed. Companies can find useful tools in CSR programs to adapt to the new reality.

KEYWORDS: Corporate Social Responsibility; Global Compact; COVID-19; Structural Change.

\section{INTRODUCCIÓN.}

La pandemia de la COVID-19 que se instauró en el mundo durante el primer semestre de 2020, ha trastornado todos los ámbitos de la vida humana. La crisis inicialmente sanitaria, 
pronto evolucionó y permeó la economía, la política, las relaciones humanas y hasta la concepción que teníamos sobre nosotros mismos como individuos. Las suposiciones sobre lo que era seguro y estable se han visto seriamente cuestionadas por la nueva enfermedad, y los planes diseñados por empresas y gobiernos deben de adaptarse rápidamente a los nuevos escenarios. Afortunadamente, no es necesario comenzar desde cero, ya que existen modelos e ideas desarrolladas hace años que trazan un camino realista hacia la recuperación, sin necesidad de regresar al status quo ante.

Aunque el concepto de Responsabilidad Social Empresarial (RSE) no es nuevo, en los últimos meses se ha iniciado en todo el mundo un repensamiento social, económico y ambiental que obliga a una reingeniería de las estrategias ante un cambio de época que requiere de nuevos enfoques. Para las empresas y organizaciones, en el futuro inmediato será más importante tomar decisiones adecuadas para su transformación y supervivencia. En este sentido, los 10 Principios del Pacto Mundial de las Naciones Unidas y los 17 Objetivos del Desarrollo Sustentable (ODS) son una guía clara de acción sobre la cual pueden basar modelos de negocios sustentables.

Conceptos como resiliencia, que la Real Academia Española define como "la capacidad humana de asumir con flexibilidad situaciones límite y sobreponerse a ellas”, (Fita, 2012, párr. 2) serán de uso común entre los empresarios, pues quienes no puedan adaptase a las nuevas condiciones post-pandemia, correrán el riego de no ser viables en el futuro. La resiliencia no es un destino; es una forma de ser. Una "organización resiliente" no es aquella que simplemente puede volver a donde se quedó antes de la crisis. Más bien, la organización verdaderamente resiliente es aquella que se ha transformado, habiendo construido las actitudes, creencias, agilidad y estructuras en su ADN que le permitan no solo regresar a donde estaba, sino catapultarse hacia adelante rápidamente (Renjen, 2020b).

El objetivo principal del presente trabajo es analizar los retos y oportunidades que tendrán las empresas en la nueva realidad post-COVID-19 y el papel que puede desempeñar la RSE para ayudar a que las empresas sobrevivan en el futuro inmediato y prosperen a largo plazo. 
Para realizar el análisis se hace una revisión de los conceptos históricos de la RSE, la influencia que han tenido el Pacto Mundial y los ODS de la ONU en la adopción de modelos de negocios sustentables y lo que proponen iniciativas de líderes mundiales como el Manifiesto de Davos 2020. Se hace una descripción del impacto económico en las empresas causada por la pandemia de la COVID-19 en el mundo y cómo a pesar de ello muchas empresas han sumado esfuerzos con gobiernos y la sociedad civil organizada para paliar los efectos negativos generados por las cuarentenas y el paro de la actividad económica. Finalmente se propone que para salir de la crisis es conveniente que los empresarios adopten modelos de negocios que sean respetuosos tanto del medio ambiente como de los derechos de los trabajadores, que se ponga énfasis en la transición de un modelo capitalista centrado en las ganancias de los accionistas (shareholders) a uno en donde se colabore con todas las partes interesadas (stakeholders) para generar progreso sustentable.

\section{EL IMPACTO DE LA COVID-19 EN LAS EMPRESAS.}

\section{II.1 La Responsabilidad Social Empresarial.}

Se tiene como precedente que en Europa y en EE.UU. se brindaron mejoras en las condiciones de vida de sus trabajadores, pero hasta pasada la Gran Depresión, de 1929 a 1933, es cuando se desarrolla en EE. UU. el Estado de Bienestar, que establece algunos compromisos en las relaciones laborales de las empresas, modificando el comportamiento asumido hasta entonces, en el que tenían como único objetivo, aumentar la productividad y sus beneficios económicos, este paradigma surge entre los años 50-60 en EE.UU. y se propaga en Europa en los 90.

A fines del siglo XX en la ciudad de Davos, Suiza, se efectuó en diciembre de 1999 una edición anual más del Foro Económico Mundial, siendo uno de los temas el relativo al impacto de la globalización. El entonces Secretario General de la Organización de las Naciones Unidas (ONU), Kofi Annan, expuso:

Hoy me complace reconocer que en los últimos dos años nuestra relación ha dado grandes pasos. Demostrándose, a través de la cooperación empresarial (...), que los 
objetivos perseguidos por las Naciones Unidas y por las compañías pueden, en efecto, alcanzarse mutuamente.

Este año, los desafío a que llevemos nuestra relación a un nivel más alto. Les propongo que ustedes, líderes empresariales reunidos en Davos, y nosotros, las Naciones Unidas, iniciemos un pacto global de valores y principios, que dará un rostro humano al mercado económico mundial (Acevedo-Guerrero et al., 2013, p. 311-312).

El Pacto Mundial 2000 nació de esta convocatoria abierta con el interés de promover la cultura de la Responsabilidad Social Empresarial (RSE).

Emanado de lo anterior se apuntalan Diez Principios universalmente aceptados que contienen la defensa y promoción en materia de derechos humanos, trabajo, medio ambiente y anti-corrupción, que gozan de consenso universal y se derivan de:

- La Declaración Universal de los Derechos Humanos.

- La Declaración de la Organización Internacional del Trabajo relativa a los Principios y Derechos Fundamentales en el Trabajo.

- La Declaración de Río sobre el Medio Ambiente y el Desarrollo.

- La Convención de las Naciones Unidas contra la Corrupción (Pacto Mundial. Red Española, 2015, párr. 1 y 2).

La Organización para la Cooperación y el Desarrollo Económico (Pacto Global. Red Chile, 2011) se incorporó al Pacto Mundial, reconociendo las directrices para las empresas multinacionales, en una gestión gremial comprometida. Los Fundamentos aludidos se promueven a nivel internacional e intentan regular la categoría de RSE, herramienta que formula criterios a seguir (Pacto Mundial. Red Española, 2015).

\section{2 ¿Qué es el Pacto Mundial?}


Está basado en la visión del antiguo Secretario General de la ONU, Kofi Annan, quien, como se expuso anteriormente, en el Foro Económico Mundial en Davos en 1999, "hizo un llamamiento a líderes empresariales para que se aliaran con la Organización para crear así un "impacto global" en cuestión de valores y principios compartidos para aportar una faceta humana al mercado global" (Kingo, s.f., párr. 3).

Actualmente, el Pacto Mundial desempeña un papel crucial en el fortalecimiento de la colaboración empresarial con las Naciones Unidas. En la actualidad, más de 11,000 empresas y más de 3,000 organizaciones, con sede en más de 160 países y más de 70 redes locales, defienden ampliamente el mensaje de que cualquier tipo de empresa, en cualquier parte del planeta, puede jugar un papel fundamental para mejorar nuestro mundo (Pacto Global, Red México, s.f.). Por su número de participantes el Pacto Global es la iniciativa corporativa más grande del mundo.

Este pacto "pide a las empresas adoptar, apoyar y promulgar, dentro de su esfera de influencia, un conjunto de valores fundamentales en las áreas de derechos humanos, normas laborales, medio ambiente y anti-corrupción” (Pacto Mundial. Red Española, 2015, párr. 3). Los países miembros de la OCDE enmiendan periódicamente las directrices para asegurar que su contenido se encuentre actualizado y refleje los cambios globales como es el caso de la emergencia sanitaria en 2020.

El propósito es que en adhesión las políticas públicas fortalezcan la confianza en empresas, trabajadores y sociedad, renueven el clima para la inversión nacional y extranjera, incrementando la contribución de las empresas al desarrollo sostenible. Los valores del Pacto Global son Cuatro, Ética, Confianza, Integridad y Transparencia, dentro de un Marco igualmente de Cuatro Elementos, con Diez Componentes qué se muestran a continuación:

\section{Tabla 1.}

Principios del Pacto Global

Marco

Derechos

Humanos

\section{Componentes}

Las empresas deben apoyar y respetar la protección de los derechos humanos fundamentales, reconocidos internacionalmente. 
Asegurarse que sus empresas no son cómplices en la vulneración de los derechos humanos.

Eliminación de la distinción o exclusión que produzca la desigualdad en el trato de solicitudes de empleo u ocupación. Libertad de afiliación y negociación.

Eliminación de toda forma de trabajo forzoso o bajo coacción.

Estándares Equivalencia en la remuneración, horas de trabajo y descanso.

Laborales

La empresa debe apoyar la erradicación de cualquier forma de trabajo infantil.

La empresa deber apoyar la abolición de las prácticas de discriminación en la ocupación y el empleo.

Las empresas deberán mantener un enfoque preventivo que favorezca el medio ambiente.

La empresa deberá fomentar las iniciativas que promuevan una mayor

Medioambiente responsabilidad ambiental.

Las empresas deberán favorecer el desarrollo y la difusión de las tecnologías respetuosas con el medioambiente.

Anticorrupción Las empresas deberán trabajar contra la corrupción en todas sus formas, incluidos extorsión y soborno.

Fuente: Acevedo-Guerrero et al., (2013), p. 312; Kingo, (s.f).

\section{3 Objetivos del Desarrollo Sustentable.}

Estos 17 objetivos globales están diseñados como una hoja de ruta para poner fin a la pobreza, proteger el planeta y garantizar que todas las personas gocen de paz y prosperidad para el año 2030. El 25 de septiembre de 2015 la Asamblea General de las Naciones Unidas adoptó de forma unánime la Agenda del Desarrollo 2030 titulada "Transformando nuestro Mundo: la Agenda 2030 para el Desarrollo Sostenible" (Organización Panamericana de la Salud, 2017). Los ODS son amplios e interdependientes, y sólo pueden llegar a cumplirse si la sociedad en su conjunto se involucra en su consecución. Muchos de los ODS están estrechamente ligados a los 10 principios del Pacto Mundial, por lo que empresas de todo el mundo han sumado sus esfuerzos para que las metas trazadas puedan cumplirse para la fecha propuesta.

Los diecisiete Objetivos del Desarrollo Sustentable se describen a continuación:

a. Erradicar la pobreza en todas sus formas en todo el mundo. 
b. Poner fin al hambre, conseguir la seguridad alimentaria y una mejor nutrición, y promover la agricultura sostenible.

c. Garantizar una vida saludable y promover el bienestar para todos y todas en todas las edades.

d. Garantizar una educación de calidad inclusiva y equitativa, y promover las oportunidades de aprendizaje permanente para todos.

e. Alcanzar la igualdad entre los géneros y empoderar a todas las mujeres y niñas.

f. Garantizar la disponibilidad y la gestión sostenible del agua y el saneamiento para todos.

g. Asegurar el acceso a energías asequibles, fiables, sostenibles y modernas para todos.

h. Fomentar el crecimiento económico sostenido, inclusivo y sostenible, el empleo pleno y productivo, y el trabajo decente para todos.

i. Desarrollar infraestructuras resilientes, promover la industrialización inclusiva y sostenible, y fomentar la innovación.

j. Reducir las desigualdades entre países y dentro de ellos.

k. Conseguir que las ciudades y los asentamientos humanos sean inclusivos, seguros, resilientes y sostenibles.

1. Garantizar las pautas de consumo y de producción sostenibles.

m. Tomar medidas urgentes para combatir el cambio climático y sus efectos.

n. Conservar y utilizar de forma sostenible los océanos, mares y recursos marinos para lograr el desarrollo sostenible. 
o. Proteger, restaurar y promover la utilización sostenible de los ecosistemas terrestres, gestionar de manera sostenible los bosques, combatir la desertificación y detener y revertir la degradación de la tierra y, frenar la pérdida de diversidad biológica.

p. Promover sociedades pacíficas e inclusivas para el desarrollo sostenible, facilitar acceso a la justicia para todos y crear instituciones eficaces, responsables e inclusivas a todos los niveles.

q. Fortalecer los medios de ejecución y reavivar la alianza mundial para el desarrollo sostenible (Naciones Unidas, 2015; Naciones Unidas, 2020).

\section{4 Manifiesto de Davos 2020.}

La última reunión del Foro Económico Mundial efectuada en noviembre de 2019, como cada año, continúa teniendo el objetivo de reunir a los principales líderes mundiales, académicos, intelectuales, informadores y más, para debatir y terceros para escuchar, sobre los problemas globales y su planteamiento para enfrentarlos.

La finalidad "marca la agenda del año sobre cómo las empresas y los gobiernos pueden hacer del mundo un lugar mejor para todos" (Pomeroy, 2020, párr. 2). Uno de los temas del cónclave, se encauzó en el propósito de las empresas en colaborar con todos sus stakeholders (empleados, clientes, proveedores, comunidades locales y la sociedad en general) y que las empresas no tengan como única meta cumplir con los intereses de sus accionistas, exhortando a considerar la creación de valor compartido y sostenido. Por su importancia se reproduce a continuación:

El mejor camino hacia la comprensión y la armonización de los intereses divergentes de todos los stakeholders es la adquisición de un compromiso común con respecto a las políticas y las decisiones que refuercen la prosperidad a largo plazo de las empresas. 
i. Una empresa cumple con sus clientes cuando les ofrece una propuesta de valor que encaja a la perfección con sus necesidades.

\section{$(\ldots)$}

ii. Una empresa trata a su personal con dignidad y respeto. (...) En un mundo sometido a constantes cambios, una empresa aboga por la continuidad en el empleo mediante la mejora continua de las competencias y la adquisición de otras nuevas.

\section{$(\ldots)$}

B. Una empresa es algo más que una unidad económica generadora de riqueza. Atiende a las aspiraciones humanas y sociales en el marco del sistema social en su conjunto.

\section{(...)}

Una empresa que opera en el ámbito multinacional no está únicamente al servicio de todos los stakeholders directamente implicados, sino que es por sí misma un stakeholder - junto con los gobiernos y la sociedad civil - de nuestro futuro global. (Pacto Global. Red México, 2020, párr. 2, 3, 7 y 8).

\section{5 La Responsabilidad Social Empresarial en México.}

Más de 700 empresas integran actualmente el capítulo México del Pacto Mundial. La actividad que desempeñan es exhortar a las demás empresas a participar y comprometerse con el Pacto Global de las Naciones Unidas e integrar Los Diez Principios y la Agenda 2030, en su actividad y crear oportunidades de acción colectiva, anteriormente citados (Kingo, 2020).

La RSE es una decisión voluntaria de las empresas por implementar los 10 Principios mencionados, que carece de una forma estandarizada de valoración. En este sentido, desde el inicio del presente siglo la adopción de los conceptos de RSE ha sido constante, aunque de manera desigual, entre los diversos sectores empresariales. A partir de la crisis sanitaria las empresas tienen la oportunidad de replantear la forma en que implementan la cultura de la RSE internamente. Para Margarita Huamán, especialista de Responsabilidad Empresarial 
de la Universidad de Castilla-La Mancha, "el impacto de la pandemia en la RSE será un impulso que llevará a la mayor adopción de una RSE positiva y transparente en su gestión, dejando de lado a una vieja RSE concentrada en marketing social y menos compromiso real” (Huamán, 2020).

Se puede observar que las empresas afiliadas a la RSE están enfrentando la situación actual en apego a los principios suscritos, evidenciando que su compromiso no representa pretensiones utópicas, ni es sólo un discurso, por el contrario, es un empeño real, permanente y voluntario a pesar de las difíciles circunstancias que imperan porque la realidad del país con el transcurso de los meses (enero a agosto de 2020) ha venido cambiando aceleradamente, de un contexto de salud controlable y breve en tiempo, a volverse en una crisis sanitaría, laboral, social, económica, incrementando el número de pobres, empeorando la violencia y discriminación a las mujeres.

\section{6 Efectos de la Pandemia de la COVID-19.}

Para mediados del mes de septiembre de 2020, el número total de contagiados reportados a nivel mundial fue de 29 millones, con más de 900 mil decesos, de acuerdo con el Centro de Recursos del Coronavirus de la Universidad Johns Hopkins. Debido a la compleja situación desencadenada por la pandemia, y al hecho de que no existe hasta el momento un tratamiento adecuado para detener la infección ni vacunas que confieran inmunidad, cada gobierno del mundo tuvo que diseñar una estrategia de contención de los contagios adecuada a su cultura, tradición o recursos económicos disponibles. Esto significó que un número importante de países tuvieran periodos de cuarentena y aislamiento durante los cuales se suspendió casi totalmente la actividad económica (Kaplan et. al., 2020). Las medidas de sana distancia, aunque menos estrictas que los cierres totales o parciales, forzaron a algunas empresas a reducir su capacidad de servicio (como en hoteles y restaurantes), a realizar trabajo remoto desde casa y efectuar gastos para cumplir con las medidas de distanciamiento e higiene (geles hidroalcohólicos, restructuración de espacios, protectores de plástico para los empleados, entre otros). (Centros para el Control y la Prevención de Enfermedades, 2020). 
Como consecuencia directa de esta situación, muchas empresas se vieron obligadas a reducir su plantilla laboral para poder sobrevivir a la súbita reducción del ingreso, y muchas otras se han declarado en quiebra. Por ejemplo, en Estados Unidos de América (el país con más muertes por el coronavirus) se estima que 1 de cada 4 restaurantes cerrarán sus puertas permanentemente (Pesce, 2020). De acuerdo con mediciones, en abril la tasa nacional de desempleo fue de $14.7 \%$, y aunque en agosto se situó en $8.4 \%$, esto significó que más de 13 millones de personas no se han reincorporado al mercado laboral estadounidense hasta septiembre (Bureau of Labor Statistics, 2020).

La crisis generada por la pandemia a nivel mundial ha tenido repercusiones económicas mucho mayores a las vislumbradas, siendo equiparable a la ocurrida en la recesión de 1930. Desde el inicio el Gobierno Federal planteó la estrategia de minimizar lo que sucedía, y transcurridos ocho meses el contagio se extendió y aumentó inesperadamente, los resultados mostraron que debería modificarse el método, el cual se sostuvo, lo que ocasionó en los diferentes sectores de la producción inestabilidad, en la pequeña, mediana y grande empresa, cerrando parcial o totalmente sus actividades. De acuerdo con la Comisión Económica para América Latina y el Caribe (CEPAL) de las Naciones Unidas, la intensidad de los efectos de la crisis, dependiendo del sector de actividad económica del que se opere, las separa en fuerte, significativa y moderada, dependiendo del sector de actividad económica del que se trate:

a. Fuerte: Servicios de turismo, industria cultural tradicional, comercio, reparación de bienes, hoteles y restaurantes, transporte, moda y automóviles.

b. Significativa: Minería, electricidad, gas y agua, construcción y materiales para la construcción, servicios empresariales, actividades financieras, bebidas, muebles y madera, industria química, electrónica-maquinaria y equipo.

c. Moderada: Agricultura, ganadería y pesca, producción de alimentos para el mercado interno, insumos y equipamiento médico, medicamentos, telecomunicaciones y envases (Comisión Económica para América Latina y el Caribe [CEPAL], 2020). 
Algunos sectores han resistido los efectos de la pandemia, incluso han experimentado cierto crecimiento. Entre ellos se encuentra el comercio electrónico (Walmart, Amazon, Mercado Libre y otras), las empresas de entrega de productos de todo tipo a domicilio (DHL, pequeños comercios y otras) y algunas grandes farmacéuticas (Pacheco, 2020). Además, otros negocios, reaccionaron modificando sus líneas de producción, distribución, principalmente alimentos, vestuario y equipo para hospitales, servicios y otros. Infortunadamente la mayoría de las pequeñas y medianas empresas han sido severamente afectadas por la crisis.

Diversos organismos internacionales advierten de las graves consecuencias económicas que tendrá la pandemia en la economía mundial. Según las previsiones del Fondo Monetario Internacional (FMI), el Producto Interno Bruto (PIB) mundial sufrirá una contracción de 4.9\% este año, causada en parte por los 11 billones de dólares (USD) que los gobiernos han gastado en un esfuerzo por detener las bancarrotas de empresas y apuntalar la economía mundial (International Monetary Fund, 2020). Las previsiones del Banco Mundial son más pesimistas, pronosticando que la caída del PIB mundial este año será del 5.2\%, la peor recesión en las últimas ocho décadas, y afectará especialmente a los mercados emergentes y países en desarrollo (World Bank Group, 2020). La pandemia de la COVID-19 ha tenido un impacto más negativo en la actividad del primer semestre de 2020 de lo previsto, y se estima que la recuperación sea más gradual, advirtió el FMI en un documento de trabajo (France24, 2020). Esto representa la recesión más profunda desde la Segunda Guerra Mundial (The World Bank, 2020), y es probable que el impacto de la COVID-19 en la economía mundial no tenga precedentes desde la Gran Depresión de la década de 1930 (He y Harris, 2020).

En este contexto adverso, las empresas mostraron un vínculo con la sociedad, reconvirtiendo sus procesos productivos a fin de dar apoyo a las diferentes necesidades en el área de la salud.

Se expone como ejemplo uno de los eventos sucedidos, como fue el caso en España. Las farmacéuticas Novartis, Sanofi y Bayer han donado medicamentos, dinero y equipamiento técnico. Empresas textiles como Mango Inditex o Pronovias reconvirtieron sus procesos a 
la fabricación de mascarillas y batas de protección del personal de hospitales, y la empresa de perfumes Garnier, del Grupo LÓréal ha distribuido miles de unidades de gel hidroalcohólico entre sus empleados y clientes (CompromisoRSE, 2020).

Más que afectar a un grupo étnico o demográfico en particular, el virus ha evidenciado y exacerbado problemas sociales como la desigualdad y pobreza. Las personas que ya se encontraban en una situación económica precaria no pueden permitirse guardar la cuarentena, ni siquiera dejar de trabajar por periodos de tiempo cortos. Muchos sólo cuentan con su ingreso diario y no están protegidos por la seguridad social, por lo que enfermarse implica un grave riesgo de que la familia padezca incertidumbre alimentaria. En Europa los datos muestran que las personas de origen africano, asiático y otras minorías tienen más probabilidades de contraer el virus y enfermarse gravemente o incluso morir por COVID-19 (He y Harris, 2020). Precisamente estos grupos se encuentran entre los más pobres y desprotegidos de las sociedades europeas. De acuerdo con David Malpass, presidente del Banco Mundial, hasta 60 millones de personas en el mundo podrían ser empujadas a la pobreza extrema como efecto directo de la pandemia (David, 2020).

Un informe reciente de la ONU muestra que la COVID-19 está teniendo impactos en prácticamente todos los ODS (United Nations, 2020). Otro grupo vulnerable son las mujeres trabajadoras, ya que constituyen una gran proporción de la fuerza laboral en sectores expuestos como el comercio minorista de alimentos y la atención de la salud, además de que en muchos países en vías de desarrollo son ellas precisamente quienes deben de cuidar a los niños, adultos mayores o familiares enfermos, lo que las expone a mayores riesgos.

Se estima que la pandemia tendrá profundos impactos económicos, sociales, políticos y culturales de largo alcance, que en conjunto ofrecen problemas, pero también son oportunidades para crear sistemas justos, incluyentes y respetuosos. Por tanto, es importante estudiar cómo podrán lidiar las empresas con el futuro incierto y qué papel jugará la Responsabilidad Social Empresarial en los planes de las corporaciones que no sucumbieron a la crisis. 
Aumento de la desigualdad, inseguridad, inestabilidad climática. ¿Es ese el futuro que queremos? Reiniciemos nuestro sistema económico. Pasemos de una economía extractiva a una regenerativa; del salario mínimo al salario digno; del descuido al respeto; dejar la mentalidad de que "el ganador se lo lleva todo" y apoyar la prosperidad compartida; cambiar del no respeto a las personas y al planeta a respetarlos. En síntesis, los imperativos para el cambio del sistema económico deben ser: bienestar compartido en un planeta saludable.

Como se mencionó, la pandemia ha dañado a la economía mundial de forma devastadora y ha puesto de manifiesto importantes vulnerabilidades en las operaciones de las empresas y las cadenas de suministro vinculadas a las condiciones de trabajo y la preparación ante desastres. Algunos de los obstáculos que presentan las compañías son: interrupciones de la cadena de suministro, problemas de flujo de efectivo, incapacidad para cumplir con las fechas de entrega, menor demanda de bienes y servicios, y afectación de la confianza de los inversionistas. Esto ha obligado a que las empresas tengan que tomar decisiones drásticas en sus modelos de negocios, lo que no ha evitado que un número importante de ellas se declare en quiebra (BBC, 2020). Entre las que resistan el impacto de las cuarentenas y la reducción de su clientela, ¿deben ser prioridad los compromisos de Responsabilidad Social Empresarial (RSE)?

\section{7 La Nueva Economía después de la pandemia.}

La Nueva Economía hace referencia al término que surgió como resultado de la transición de una economía basada en la manufactura a una basada en el conocimiento y la tecnología, especialmente la digital. Este concepto, surgido a fines del siglo pasado, plantea pasar de un modelo de producción obsoleto, basado en el consumo del carbón y derivados de los hidrocarburos, a uno en donde las energías limpias, como la solar, eólica e hídrica, sean los pilares que muevan la economía. (Goodell, 2019; López, 2018).

En este sentido, este tipo de desarrollo económico, eminentemente sustentable, se alinea con los objetivos planteados por la ONU en sus diversos planes de desarrollo, muchos de los cuales influyen de manera directa las directrices de la RSE moderna. En un momento en 
el que se cuestiona la viabilidad de los ODS debido a la crisis actual (Nature Editorial, 2020), diversos economistas como Jeremy Rifkin, Thomas Piketty o Jeffrey D. Sachs han defendido que se redoblen los esfuerzos por lograr alcanzar los objetivos planteados y aceptados por la comunidad internacional (Sachs et al., 2020), así como políticos europeos (Pellicer, 2020). Especialmente, ponen de manifiesto que el planeta no puede esperar más y que continuar utilizando energías no renovables es un camino sin retorno para la economía mundial. En vez de ver a la actual crisis como un factor que impedirá alcanzar los objetivos deseados, quienes apoyan la reconversión energética por combustibles verdes apuntan a que es posible cambiar y de manera rápida los hábitos de consumo de la sociedad, como ha quedado demostrado en meses recientes.

Para Rifkin, uno de los principales promotores del proyecto denominado Green New Deal (haciendo referencia a la serie de programas implementados por el presidente estadounidense Franklin D. Roosevelt en la década de 1930 para sacar a su país de la Gran Depresión) una nueva visión para el futuro está ganando impulso rápidamente. Frente a la emergencia global causada por el cambio climático se propone una agenda para un movimiento político con el potencial de revolucionar la economía mundial en los años venideros. Es importante recalcar que quienes están al frente de este nuevo proyecto sustentable no son los políticos, sino la sociedad civil organizada $\mathrm{y}$, cada vez con mayor frecuencia, la comunidad empresarial (Rifkin, 2019).

Sectores clave de la economía se están desvinculando rápidamente de los combustibles fósiles en favor de energías renovables cada vez más baratas y las nuevas oportunidades de negocio y empleo que las acompañan. Esto generará una burbuja de activos de carbono de varios billones de dólares que se quedarán varados en la nueva economía (las plataformas petroleras, refinerías, oleoductos, etc.), y seguir apostando por energías no renovables sin duda afectará las proyecciones de crecimiento de las empresas y gobiernos que se resistan al cambio (Rifkin, 2019).

El Green New Deal abre la posibilidad de un cambio masivo hacia una era ecológica postcarbono que evite un aumento de la temperatura del planeta que nos llevará al borde del cambio climático descontrolado. El crecimiento que esta nueva economía generará 
posiblemente no sea exponencial como lo ha sido durante la era del petróleo, pero sin duda será sostenible. Ya no es posible que el capitalismo continúe como hasta la actualidad, pero las empresas que se sumen a esta visión ecológica estarán desarrollando un nuevo tipo de capitalismo que se intersecta con las demandas sociales de mejor distribución del ingreso, respeto a los derechos humanos de todos y todas y sustentabilidad ecológica.

Estas propuestas están encontrando eco en diversos foros, el más importante de ellos el Foro Económico Mundial. En el mes de junio de este año, algunos de los líderes empresariales más importantes del mundo, así como mandatarios y activistas se reunieron de manera virtual para anunciar una propuesta denominada The Great Reset [el Gran Reinicio], la cual aboga por la adopción de políticas como el aumentar impuestos a los estratos más ricos de la sociedad, mayor regulación de los mercados y adopción por parte de los gobiernos de proyectos como el Green New Deal (Schwab y Malleret, 2020a; Schwab y Malleret, 2020b).

De acuerdo con Klaus Schwab, fundador y presidente ejecutivo del Foro Económico Mundial, deben participar todos los países, desde los Estados Unidos hasta China, y deben transformarse todos los sectores, desde el gas y el petróleo hasta el de la tecnología, En resumen, se necesita un "gran reinicio" del capitalismo. Para Schwab todos los aspectos de nuestras sociedades y economías deben ser renovados, desde la educación hasta los contratos sociales y las condiciones laborales (Schwab, 2020).

La RSE permite que las empresas articulen sus políticas de gobernanza interna y su modelo de negocio hacia la consecución de estos objetivos sustentables. Hacerlo les permitirá apoyar los esfuerzos de la sociedad por realizar un cambio sustentable a favor de nuestro planeta.

\section{METODOLOGÍA, TÉCNICAS Y MATERIALES EMPLEADOS.}

Para el desarrollo de este trabajo se utilizaron fuentes diversas de información como textos de investigación, medios electrónicos, revistas especializadas en negocios y salud, y diarios de circulación nacional e internacional. Es una investigación de análisis cualitativo. Se empleó el método deductivo para la fundamentación soportada en juicios argumentativos y 
debatibles, con respaldo en diversas fuentes informativas que, por su actualidad, permiten exponer la vigencia del problema.

\section{PRINCIPALES HALLAZGOS.}

Invertir más en esquemas que promuevan y refuercen la RSE, incrementando el compromiso social en aras de afrontar los retos económicos a corto y largo plazo. Existen actualmente empresas que dejan de invertir en RSE debido a la necesidad de concentrarse en su negocio operativo para sobrevivir, y también casos de empresas que han actuado sin ética con la finalidad de evitar los cierres por cuarentena. Otras han inflado precios de bienes esenciales, realizando afirmaciones engañosas sobre los productos que venden, como los farmacéuticos (He y Harris, 2020).

Sin embargo, diversos estudios demuestran que la gran mayoría de las empresas durante la crisis no sólo han mantenido sus compromisos de RSE, rechazando las prácticas comerciales poco éticas, sino que han contribuido proactivamente a los esfuerzos nacionales para combatir la enfermedad, poniendo a disposición de la sociedad recursos, tanto financieros como materiales (Galante, 2020).

Es posible que la pandemia acelere el desarrollo de la RSE a largo plazo, ya que cada vez más empresas y negocios se dan cuenta de que su supervivencia y desarrollo depende de lograr un delicado equilibrio entre rentabilidad y armonía con sus diversos grupos de interés (stakeholders). (Hagel et al., 2020). Si algo ha demostrado esta pandemia es que la participación activa de la sociedad en su conjunto es clave para frenar los contagios y ayudar a que la economía se recupere de manera rápida, lo que sin duda aumentará la expectativa de la gente de que las empresas sean más responsables socialmente. Por lo tanto, podemos imaginar el período posterior a la pandemia como uno en el que las empresas prósperas son aquellas con un fuerte compromiso y estrategias de RSE efectivas e implementaciones eficientes. (He y Harris, 2020).

De acuerdo con la OCDE: 
Es probable que las empresas que tomen medidas proactivas para abordar los riesgos relacionados con la crisis de COVID-19 de una manera que mitigue los impactos adversos sobre los trabajadores y las cadenas de suministro generen más valor y resiliencia a largo plazo, mejorando su viabilidad a corto plazo y sus perspectivas de recuperación tanto en el mediano como largo plazo. (OECD, 2020).

Un ejemplo de vínculo con la sociedad es España. De acuerdo con un análisis de cerca de 200 iniciativas empresariales de más de 100 empresas con operación en dicho país, los programas de RSE se han orientado a fin de ser parte de la solución a esta crisis. Entre las 15 principales tendencias en RSE están: acciones para la prevención; donación de material sanitario, donaciones de dinero o en forma de productos y servicios de la empresa; impulso de campañas de recaudación de fondos; reconversión de la industria para la fabricación de material sanitario; infraestructura operativa y logística al servicio de la sociedad; flexibilidad en los plazos y condiciones de pago; líneas de crédito contra la crisis; atención prioritaria a adultos mayores, mujeres embarazadas, personas con discapacidad y personal médico; digitalización de servicios; compromiso por el mantenimiento de puestos de trabajo; bonos salariales a empleados sometidos a fuertes jornadas laborales; flexibilidad laboral para conciliar; reducción del plazo de pago a proveedores (PYMES y autónomos); reducción de ingresos anuales de altos directivos y desarrollo de nuevas alianzas, públicas y privadas, más allá de la competencia (Galante, 2020).

Se recomienda a las empresas incluir acciones concretas como las que se describen a continuación:

a. Servir de enlace con los equipos de adquisiciones y sostenibilidad para comprender las vulnerabilidades de los proveedores o socios comerciales y, en particular, el desempeño del proveedor en las relaciones laborales y los problemas de salud y seguridad.

b. Desarrollar un plan de diligencia debida ante interrupciones críticas de suministros del proveedor, en estrecha consulta con los equipos de gestión de adquisiciones, sostenibilidad o ambiental y social de la empresa, para conseguir nuevas fuentes o 
proveedores, centrando las evaluaciones de proveedores en los problemas de RSE más importantes, que podrían afectar la planificación de contingencias a corto y largo plazo.

c. Evaluar los impactos de los pedidos cancelados o suspendidos en el negocio, incluidos sus impactos ambientales o sociales, y la capacidad de reiniciar después de la crisis.

d. Considerar soluciones innovadoras, como recortes en los sueldos de los ejecutivos o cancelación de pagos de dividendos (si es relevante), para ayudar a financiar los salarios y evitar despidos o licencias.

e. Al considerar suspender, cancelar o reducir temporalmente los pedidos u otros tipos de negocios con proveedores y socios comerciales, evaluar los impactos socioeconómicos de varias opciones de contingencia, teniendo en cuenta la disponibilidad de cualquier préstamo o paquete de ayuda de emergencia y apoyo. Consultar, si es posible, con los trabajadores o sus representantes a nivel global (por ejemplo, sindicatos globales) sobre las opciones de contingencia y comunicar sus respectivos impactos a los gobiernos. Buscar el apoyo de donantes u otra ayuda para mitigar los efectos socioeconómicos de la cancelación de negocios. (OCDE, 2020).

La crisis despertó en los clientes que la demanda que hacen tenga la calidad de los insumos y los precios sean accesibles para su consumo. Las empresas deben adoptar modelos flexibles, innovadores, creativos y orientados a la rentabilidad sostenible. Debido a que una de las consecuencias de las varias cuarentenas han sido los despidos masivos, esto ha ocasionado que los consumidores estén atentos en conocer qué empresas han hecho lo conveniente para mantener a sus empleados y también estarán atentos en conocer qué empresas actuaron en contario, eliminado puestos de trabajo (Renjen, 2020a).

El concepto resiliencia debe de formar parte del vocabulario de los líderes empresariales. La Firma Deloitte considera cinco cambios importantes en el entorno empresarial: 
a. Cambios en el contrato social. El contrato implícito entre las empresas y sus partes interesadas está cambiando para garantizar la viabilidad de todos. Las nuevas consideraciones en torno al equilibrio entre el trabajo y el bienestar de los empleados sugieren que estos factores están remodelando un nuevo estándar en la forma en que las empresas funcionan.

b. Cambios en los roles y reglas de las instituciones. La crisis ha mostrado la fluidez entre los sectores público y privado, y ha hecho que Organizaciones No Gubernamentales (ONG) y otras agencias entren de lleno en la esfera pública. Al mismo tiempo, las asociaciones público-privadas muestran una nueva forma de cooperación que difumina las fronteras públicas/privadas tradicionales y puede presagiar una mayor colaboración entre el gobierno y las empresas.

c. Impredecibilidad en fuentes y usos de financiamiento y en los mercados de capitales. Debido al impacto económico de la pandemia las fuentes de efectivo, su uso y la propia liquidez han sido impredecibles. Las empresas deberán planificar variaciones en su situación financiera y evaluar su capacidad para manejar una posible carga de deuda creciente.

d. Permanencia de los cambios de comportamiento del cliente. Como ya se mencionó en párrafos anteriores, la crisis ya ha tenido un impacto profundo en el comportamiento de los clientes. Las empresas deberán anticipar si la pandemia ha alterado permanentemente los comportamientos, experiencias, expectativas y el papel de la participación digital de sus clientes y de qué manera.

e. Expectativas de seguridad física, emocional, financiera y digital. La incertidumbre dará paso a temores entre las partes interesadas, por lo que las empresas deben de comprender cómo las expectativas de seguridad y protección han cambiado, quizás de forma permanente (Renjen, 2020b).

Las empresas éticamente alineadas a la RSE durante esta crisis se distinguieron por apegarse a sus cuatro principios: 
a. El negocio, el cual debe ser eficiente, responsable, resiliente y de calidad;

b. Los trabajadores, no solo desde el punto de vista de mantener los salarios, sino de su salud y bienestar;

c. Los clientes, con los cuales se ha tenido que fortalecer su relación y vínculos; y

d. La sociedad, para poder aportar soluciones al bienestar común (Canal, 2020).

\section{CONCLUSIONES.}

El cambio en las condiciones sanitarias (31 de octubre de 2020) similar al que está ocurriendo en países europeos como España, Francia y Alemania inducirá a aumentar el deterioro económico en el país, situación factible que suceda por las características similares a la de esos países, vislumbrándose un escenario incierto. El incipiente crecimiento económico vaticinado, se vería interrumpido, con un deterioro en las expectativas de 2021.

La participación de empresarios con afinidad en la Responsabilidad Social Empresarial será transcendente en que logren generar confianza, primero en la parte interna, empleados, proveedores, accionistas y otras, y posteriormente la parte la externa, clientes, acreedores, bancos y otras, generando una alternativa en el número de consumidores, que se reflejará en incremento de ventas, ventaja con la competencia y mayor oportunidad de permanencia en el mercado. Tener una visión clara de hacia dónde se quieren llegar, cómo y cuándo alcanzarlo, infunde confianza y seguridad.

Los conceptos de sustentabilidad ecológica y los proyectos refundadores del capitalismo del siglo XXI son oportunidades que los empresarios con visión puedan desarrollar. El cambio hacia una cultura que respete el medio ambiente y a las personas está en marcha, liderado por las generaciones jóvenes. Los jóvenes consumidores, más conscientes del impacto que tienen en la economía, comienzan a demandar a las empresas que actualicen su forma de producción. La recuperación económica pasará por un replanteamiento de la 
función que la propia empresa tiene que desarrollar en favor de la sociedad. No se regresará a la economía pre-COVID-19.

Las empresas que se apliquen al cambio y se apremien a transformarse e innovar su modelo de negocio, serán quienes continuarán en el corto y largo plazo. La RSE tomará un papel substancial en la reconfiguración del nuevo orden económico de las prioridades empresariales, de las que dependerá la economía global. La sociedad civil desarrollará y emprenderá un mayor activismo en las demandas y participación social en 2021.

\section{REFERENCIAS.}

Acevedo-Guerrero, J. A., Zárate-Rueda, R. y Garzón-Ruiz, W. F. (2013). Estatus jurídico de la Responsabilidad Social Empresarial (RSE) en Colombia. Díkaion, 22(2) 303-332.

BBC. (30 de julio de 2020). Coronavirus: US economy sees sharpest contraction in decades. https://www.bbc.com/news/business-53574953

Bureau of Labor Statistics (4 de septiembre de 2020). The Employment Situation-August 2020. U.S. Department of Labor. https://www.bls.gov/news.release/pdf/empsit.pdf

Canal, R. (26 de junio de 2020). COVID-19: Una prueba real de responsabilidad social. Deloitte. https://www2.deloitte.com/mx/es/pages/dnoticias/articles/COVID-19-una-prueba-deresponsabilidad-social.html

Centros para el Control y la Prevención de Enfermedades. (15 de julio de 2020). Enfermedades del $\begin{array}{llll}\text { coronavirus } & 2019 \quad \text { (COVID-19): }\end{array}$ https://espanol.cdc.gov/coronavirus/2019-ncov/prevent-getting-sick/social-distancing.html

Comisión Económica para América Latina y el Caribe. (2 de julio de 2020). Sectores y empresas frente al COVID-19: Emergencia y reactivación. Informe Especial COVID-19 No. 4. https://repositorio.cepal.org/bitstream/handle/11362/45734/4/S2000438_es.pdf

CompromisoRSE. (21 de abril de 2020). COVID-19, la crisis sanitaria, económica y social que pone a prueba la RSE. https://www.compromisorse.com/RSE/2020/04/21/COVID-19-LACRISIS-SANITARIA-ECONOMICA-Y-SOCIAL-QUE-PONE-A-PRUEBA-LA-RSE/

David, D. (7 de junio de 2020). Coronavirus 'a devastating blow for world economy'. BBC News. https://www.bbc.com/news/business-52939846

Fita, J. (20 de noviembre de 2012). ¿Eres resiliente? La Vanguardia.

https://www.lavanguardia.com/vida/20121120/54355244814/eres-resiliente.html

France24. (24 de junio de 2020). Covid-19 sparks global economic 'crisis like no other', IMF says. https://www.france24.com/en/20200624-imf-says-covid-19-sparks-global-economic-crisislike-no-other

Galante, E. (abril de 2020). 15 tendencias en Responsabilidad Social Corporativa ante el COVID19. Ethic. https://ethic.es/2020/04/tendencias-responsabilidad-corporativa-covid19/ 
Goodell, J. (17 de septiembre de 2019). Is the Green New Deal realistic? Two sympathetic authors weigh in. The New York Times. https://www.nytimes.com/2019/09/17/books/review/on-firegreen-new-deal-naomi-klein.html

Hagel, J., Jacobson, S. y Brown, J. S. (3 de junio de 2020). New architectures of resilience. Are you heading for a restart or a new start? Dolitte Insights.

https://www2.deloitte.com/global/en/insights/economy/covid-19/post-covid-restart-or-new$\underline{\text { start.html }}$

He, H. y Harris, L. (2020). The impact of Covid-19 pandemic on corporate social responsibility and marketing philosophy. Journal of Business Research, 116, 176-182. https://doi.org/10.1016/j.jbusres.2020.05.030

Huamán, S. M. (2 de junio de 2020). Impacto del COVID-19 en la Responsabilidad Social Empresarial: Una Mirada desde Latinoamérica. SIRSE. http://sirse.info/impacto-delcovid-19-en-la-responsabilidad-social-empresarial-una-mirada-desde-latinoamerica/

International Monetary Fund. (20 de junio de 2020). World Economic Outlook Update. https://www.imf.org/en/Publications/WEO/Issues/2020/06/24/WEOUpdateJune2020

Kaplan, J., Frías, L y McFall-Johnsen, M. (25 de agosto de 2020). Our ongoing list of how countries are reopening, and which ones remain under lockdown. Business Insider.

https://www.businessinsider.com/countries-on-lockdown-coronavirus-italy-2020-3?r=MX\&IR=T

Kingo, L. (s.f.). El Pacto Mundial de la ONU: La búsqueda de soluciones para retos globales. Naciones Unidas. https://www.un.org/es/cr\%C3\%B3nica-onu/el-pacto-mundial-de-la-onula-b\%C3\%BAsqueda-de-soluciones-para-retos-globales

Kingo, L. (17 de marzo de 2020). Uniendo a las empresas a través de los Diez Principios para afrontar el COVID-19. Pacto Global. Red México.

https://www.pactomundial.org.mx/2020/03/17/uniendo-a-las-empresas-a-traves-de-los-diezprincipios-para-afrontar-el-covid-19/

López, A. (17 de abril de 2018). ¿Thomas Pikkety, Jeffrey Sachs y Jeremy Rifkyn en la Colombia Humana? Al Poniente. https://alponiente.com/thomas-pikkety-jeffrey-sachs-y-jeremyrifkyn-en-la-colombia-humana/

Naciones Unidas. (2015). Memoria del Secretario General sobre la labor de la Organización. https://undocs.org/es/A/70/1

Naciones Unidas. (2020). Informe de los Objetivos de Desarrollo Sostenible 2020. https://unstats.un.org/sdgs/report/2020/The-Sustainable-Development-Goals-Report2020_Spanish.pdf

Nature Editorial. (2020). Time to revise the Sustainable Development Goals. Nature, 583, 331-332. doi:10.1038/d41586-020-02002-3

Organización para la Cooperación y el Desarrollo Económicos. (2020). COVID-19 y la Conducta Empresarial Responsable. OECD Publishing. https://read.oecdilibrary.org/view/?ref=130_130612-yej4uhtct8\&title=El-COVID-19-y-la-Conducta-

Empresarial-Responsable

Organización Panamericana de la Salud. (Mayo de 2017). Información Regional de la Implementación de la Agenda 2030 para el Desarrollo Sostenible en la Región de las Américas. https://www.paho.org/hq/dmdocuments/2017/sdgs-briefing-on-spa.pdf

Pacheco, R. (16 de octubre de 2020). Historias de éxito en la crisis. Dinero en Imagen. 
https://www.dineroenimagen.com/rodrigo-pacheco/historias-de-exito-en-lacrisis $/ 127945 ? \mathrm{mc}$ cid $=8050 \mathrm{afafbd} \& \mathrm{mc}$ eid $=\operatorname{dc} 636 \mathrm{cf} 900$

Pacto Global. Red Chile. (2011). El Pacto Global y la OCDE: Hacia una nueva agenda de colaboración. $\quad$ https://pactoglobal.cl/2011/el-pacto-global-y-la-ocde-hacia-una-nuevaagenda-de-colaboracion/

Pacto Global. Red México (s.f.). ¿Qué es el Pacto Mundial? https://www.pactomundial.org.mx/que-es-el-pacto-mundial/

Pacto Global. Red México. (17 de abril de 2020). Manifiesto de Davos 2020. https://www.pactomundial.org.mx/2020/04/17/manifiesto-de-davos-2020-2/

Pacto Mundial. Red Española. (18 de febrero de 2015). Los 10 Principios del Pacto Mundial. www.pactomundial.org/2015/02/10-principios-del-pacto-mundial

Pellicer, L. (16 de septiembre de 2020). Von der Leyen redobla su apuesta por la "agenda verde" para la reconstrucción económica y social de la UE. El País.

https://elpais.com/internacional/2020-09-16/von-der-leyen-fija-ante-la-eurocamara-las-prioridadesde-un-nuevo-curso-marcado-por-la-pandemia.html

Pesce, N. L. (15 de mayo de 2020). 1 in 4 restaurants won't reopen after the coronavirus pandemic, OpenTable CEO warns. Market Watch.

https://www.marketwatch.com/story/1-in-4-restaurants-wont-reopen-after-the-coronaviruspandemic-opentable-ceo-warns-2020-05-15?mod=article_inline

Pomeroy, R. (20 de enero de 2020). ¿Cuál es la realidad en Davos, qué es lo que realmente sucede allí Expansión. https://expansion.mx/opinion/2020/01/20/cual-es-la-realidad-en-davosque-es-lo-que-realmente-sucede-alli

Renjen, P. (16 de marzo de 2020a). The heart of resilient leadership: Responding to COVID-19. A guide for senior executives. Deloitte Insights.

https://www2.deloitte.com/global/en/insights/economy/covid-19/heart-of-resilient-leadershipresponding-to-covid-19.html

Renjen, P. (22 de abril de 2020b). The essence of resilient leadership: Business recovery from COVID-19. Dolitte Insights. https://www2.deloitte.com/global/en/insights/economy/covid19/guide-to-organizational-recovery-for-senior-executives-heart-of-resilient-leadership.html

Rifkin, J. (2019). The Green New Deal: Why Fossil Fuel Civilization Will Collapse by 2028 and the Bold Economic Plan to Save Life on Earth. St. Martin's Press.

Sachs, J. D., Schmidt-Traub, G. y Lafortune, G. (11 de septiembre de 2020). Hay que decirle la verdad al poder sobre los ODS. El País.

https://elpais.com/elpais/2020/09/09/planeta_futuro/1599656859_893889.html

Schwab, K. (3 de junio de 2020). Ahora es el momento de un 'gran reinicio'. World Economic Forum. https://es.weforum.org/agenda/2020/06/ahora-es-el-momento-de-un-gran-reinicio/

Schwab, K. y Malleret, T. (2020a). COVID-19: The Great Reset. Forum Publishing.

Schwab, K. y Malleret, T. (14 de julio de 2020b). COVID-19's legacy: This is how to get the Great Reset right. World Economic Forum. https://www.weforum.org/agenda/2020/07/covid19this-is-how-to-get-the-great-reset-right

The World Bank (8 de junio de 2020). COVID-19 to Plunge Global Economy into Worst Recession since World War II. https://www.worldbank.org/en/news/press-release/2020/06/08/covid19-to-plunge-global-economy-into-worst-recession-since-world-war-ii 
United Nations. (7 de julio de 2020). UN report finds COVID-19 is reversing decades of progress on poverty, healthcare and education. UN Department of Economic and Social Affairs. https://www.un.org/development/desa/en/news/sustainable/sustainable-development-goalsreport-2020.html

World Bank Group. (Junio de 2020). Global Economic Prospects. International Bank for Reconstruction and Development/The World Bank.

https://openknowledge.worldbank.org/bitstream/handle/10986/33748/9781464815539.pdf?sequence $=20$ \&isAllowed $=\mathrm{y}$ 